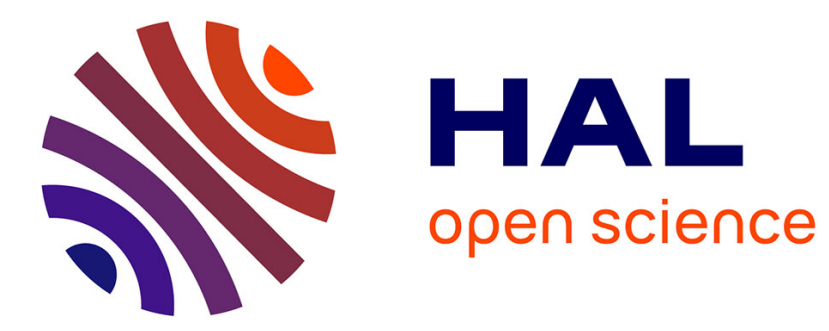

\title{
Experimental observation of minigap stripes in periodically corrugated broad photonic wires
}

Omer Khayam, Henri Benisty, Cyril Cambournac

\section{To cite this version:}

Omer Khayam, Henri Benisty, Cyril Cambournac. Experimental observation of minigap stripes in periodically corrugated broad photonic wires. Physical Review B: Condensed Matter and Materials Physics (1998-2015), 2008, 78 (15), pp.153107. 10.1103/PhysRevB.78.153107 . hal-00567028

\section{HAL Id: hal-00567028 \\ https://hal-iogs.archives-ouvertes.fr/hal-00567028}

Submitted on 5 Apr 2016

HAL is a multi-disciplinary open access archive for the deposit and dissemination of scientific research documents, whether they are published or not. The documents may come from teaching and research institutions in France or abroad, or from public or private research centers.
L'archive ouverte pluridisciplinaire HAL, est destinée au dépôt et à la diffusion de documents scientifiques de niveau recherche, publiés ou non, émanant des établissements d'enseignement et de recherche français ou étrangers, des laboratoires publics ou privés. 


\title{
Experimental observation of minigap stripes in periodically corrugated broad photonic wires
}

\author{
O. Khayam, H. Benisty, and C. Cambournac \\ Laboratoire Charles Fabry de l'Institut d'Optique, CNRS, Université Paris-Sud, Campus Polytechnique, \\ RD 128, 91127 Palaiseau Cedex, France \\ (Received 22 February 2008; revised manuscript received 4 July 2008; published 20 October 2008)
}

\begin{abstract}
A multimodal one-dimensional photonic wire subject to a periodic corrugation of the boundary exhibits minigaps among its dispersion branches. For equidistant branches, these minigaps form marked stripes that occupy a substantial fraction of the dispersion diagram and deeply modulate the photon density of states. We experimentally observe these stripes in relatively broad planar photonic wires-15-30 $\lambda / n$ wide-whose lateral confinement is based on two-dimensional photonic-crystal lattices defined in a vertically confining $\mathrm{InP}$ heterostructure. The waveguides are tilted at an angle to collect the spontaneous emission of embedded quantum wells channeled into the modes corresponding to the dispersion branches. The minigap stripes manifest themselves as large intensity modulations in the spontaneous emission spectra in TE and TM polarizations. An analogy with the appearance of similar minigap stripes in the recently studied edge-corrugated graphene nanoribbons is pointed out.
\end{abstract}

DOI: 10.1103/PhysRevB.78.153107

A photonic wire ${ }^{1}$ is the simplest extended lowdimensional system freezing two of the three photon degrees of freedom. A periodic perturbation along the wire causes mode coupling and depending upon the nature of the perturbation "minigaps"2 may appear at the mode crossings away from the Brillouin-zone (BZ) edge. As a result, the distribution of the one-dimensional (1D) photon density of states (DOS) in the wire is drastically modified. The subsequent DOS singularities have been shown to enhance spontaneous emission (SpE) (Refs. 3-5) and gain ${ }^{6}$ and to slow down light, ${ }^{7}$ all this with the advantage of an open-cavity configuration. For instance, a recent proposal of a "single-photon gun" 8 exploits the large Purcell effect in short periodic wires.

In this Brief Report, we study a phenomenon originating from the multimode nature of broad periodic wires that strongly patterns the 1D photon DOS, namely, stripes of minigaps that cause the dispersion bands to roll up like

PACS number(s): 42.70.Qs, 42.50.Xa, 42.82.Et

"necklaces" in the wave-vector space ( $k$ space). Broad periodic wires have been studied lately in various contexts, ${ }^{2,4,6,9-11}$ but such a DOS modulation has neither been recognized nor experimentally reported. In a standard waveguide geometry, the optical guided modes corresponding to these dispersion bands remain trapped because of total internal reflection (TIR). Here, we propose an original technique successfully evidencing the modulated DOS in periodically corrugated broad wires in both TE and TM polarizations of light.

To guide the reader in the maze of photonic semiconductor structures, we refer to Fig. 1. A basic 1D dielectric wire of cross-section $\mathrm{L}_{\mathrm{x}} \times \mathrm{L}_{\mathrm{y}}$ (core refractive index $n_{2}$ and cladding index $n_{1}<n_{2}$ ) is shown in Fig. 1(a). The optical field is confined in the $x y$ plane, and propagation wave vector is defined along the $z$ direction. In Fig. 1(b), with an added periodicity " $a$ " along $z$, such a wire is akin to the common (a)

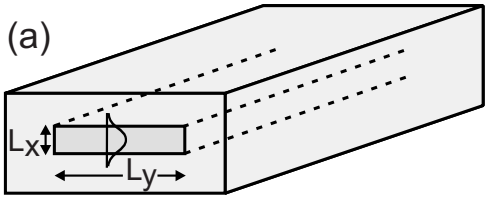

$\sim \zeta(\mathrm{x}) \psi(\mathrm{y}) \exp \left(\mathrm{ik}_{\mathrm{z}} \mathrm{z}\right)$

(b)
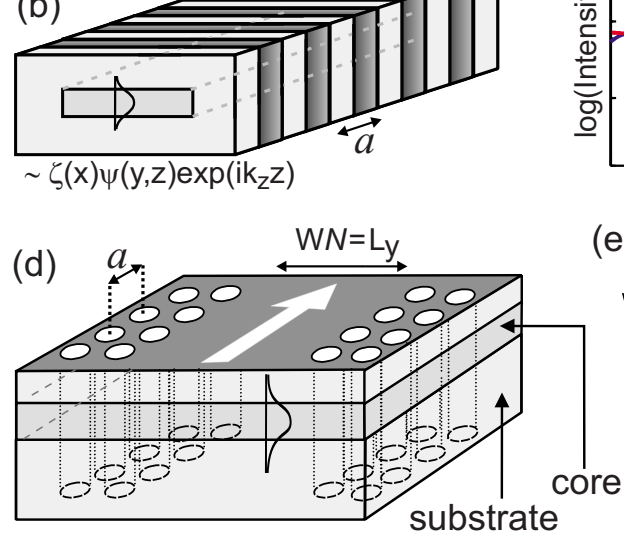

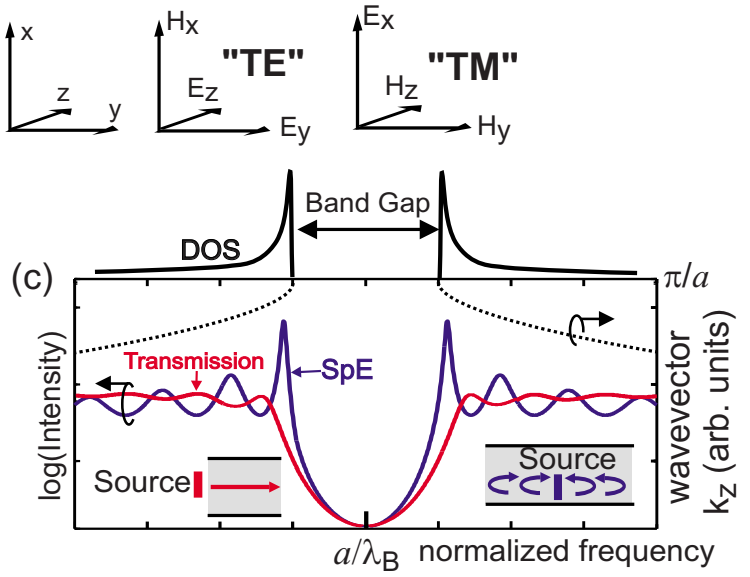

(e)

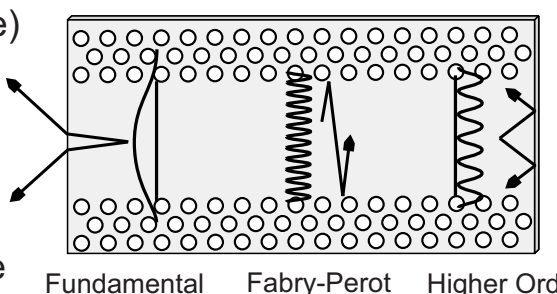

FIG. 1. (Color online) (a) Simple dielectric photonic wire. (b) Wire with 1D periodicity. (c) The SpE and transmission spectra envelope of such a periodic wire reflect its DOS profile. [(d)-(e)] Wire with $1 \mathrm{D}$ periodicity from $2 \mathrm{D}$ $\mathrm{PhC}$ confinement along with its various transverse modes. 
distributed feedback (DFB) structure of laser diodes. Modes are subject to longitudinal feedback and a band gap opens only at the crossing of the dispersion branches at the first BZ edge $\left(k_{\mathrm{z}}=\pi / a\right)$, as shown by the dotted lines in Fig. 1(c).

The SpE spectrum of such DFB laser diodes [Fig. 1(c)] is a classical example of emission inside a $1 \mathrm{D}$ periodic structure. ${ }^{12}$ The band gap inhibits emission, and the resulting spectrum envelope reproduces the DOS of the 1D system. The relationship between the transmission of a periodic system and the SpE due to an embedded source, both collected at its exit, can be inferred from Fig. 1(c). The transmission exhibits weaker modulations than the $\mathrm{SpE}$ spectrum and is notably deprived of the "overshoot" related to the 1D DOS divergence at the band edge. For instance, transmission has a cusp rather than a divergence at gap edges. It is therefore advantageous and interesting to study $\mathrm{SpE}$ from the inside of such wires rather than probing their modal transmission.

The wires studied in this Brief Report basically have the same ingredient-1D periodicity along the propagation direction. However, the lateral confinement is provided by a two-dimensional (2D) photonic-crystal (PhC) lattice, as shown in Fig. 1(d). Such waveguides are widely studied in the regime $L_{\mathrm{y}} \geqslant L_{x}$ and are labeled in terms of their canonical width "WN," where $N$ is the number of missing rows of holes along the $\Gamma K$ direction of a triangular lattice of air holes. For a large aspect ratio $L_{y} / L_{x}$ and monomode vertical confinement, a top view suffices. Figure 1(e) shows the top view of the broad $\mathrm{PhC}$ wire with transverse-mode profiles and their associated rays based on the simple wave-vector angle equivalence $k_{\mathrm{z}}=2 n u \sin \theta$, where $u=a / \lambda$ is the normalized frequency, $n$ is the index of the wire core, and $\theta$ is the propagation angle in the core. For high indices $(n \sim 3)$ the critical angle at the dielectric-air interface is only $\sim 20^{\circ}$, and thus, most of the modes do not escape out of the waveguide into the air. Figure 1(e) depicts this situation where a higher-order mode (HOM) is back-reflected into the waveguide at the interface and thus remains unobserved on the outside. It is this type of mode on which we shall observe a strong SpE modulation effect.

The periodicity along the wire evokes the Bloch-Floquet theorem, and a supercell plane-wave expansion ${ }^{13}$ can be applied to obtain the band structure. Figure 2 shows the dispersion diagram of a $W 31 \mathrm{PhC}$ wire extended to the second $\mathrm{BZ}$ to reveal symmetry. The dispersion branches around the first BZ edge appear as "chain necklaces" separated by stripes of minigaps occupying a large fraction of the $k$ space. Such structuring of the photonic bands, and hence the 1D photon DOS, originates from the regularity of the dispersion branches in conjunction with the intermodal coupling and can be understood as follows. The periodic corrugation of the wire boundary couples two contrapropagating guided modes and forms a minigap locally at their crossing in $k$ space. In the case of mode branches following a hyperbolic dispersion relation, ${ }^{14}$ adjacent minigaps merge together forming stripes that obey themselves a similar hyperbolic function centered, however, at $k_{\mathrm{z}}=\pi / a$. Adding a parity selection rule to the modal interaction scheme (canceling even-odd mode coupling) results in the equidistant necklacelike aspect of Fig. 2.

The pronounced $k$ space modulation of the photon DOS brought about by the minigap stripes is unique to multimode

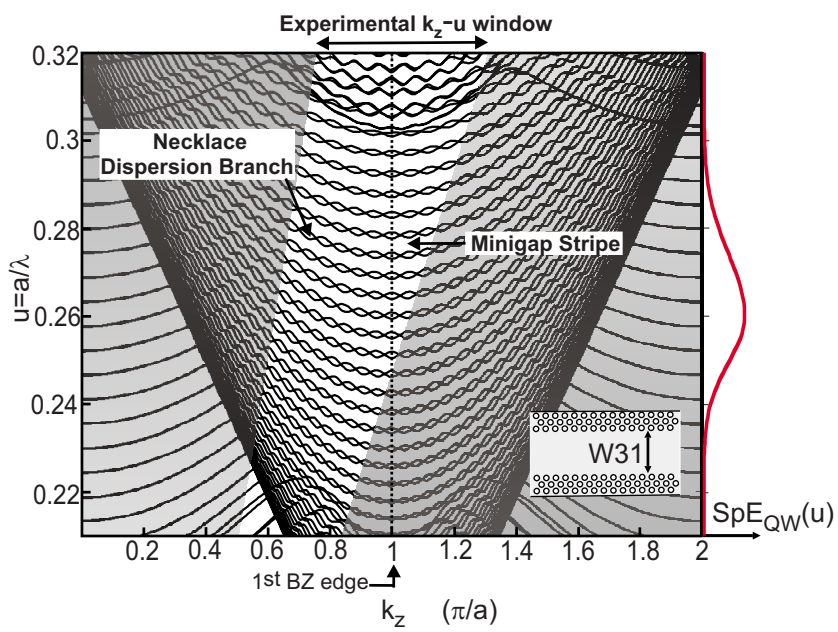

FIG. 2. (Color online) Dispersion diagram of a W31 waveguide (scheme in the inset) for TE polarization. The unshaded region is the experimental $k_{\mathrm{z}}-u$ window. The embedded QW bare luminescence is indicated in red.

periodically corrugated wires. At the first BZ edge, the frequency spacing of the equidistant necklace branches, i.e., the free spectral range (FSR), scales inversely with $W N$. The ratio of minigap stripe width to stripe FSR only depends on the nature of the photonic crystal and not on the guide width. ${ }^{15}$ For the present $\mathrm{PhC}$ case (air filling factor $f=35 \%$ and $n=3.21$ as we shall see), without special design, minigap stripes turn out to be wider than the necklace-paired branches. This DOS modulation of the wire shall manifest itself as a modified $\mathrm{SpE}$ spectrum of a source placed inside it, as depicted in Fig. 1(c), and shall be observed in the experiments as detailed later below.

An analogy with electron states exists in the band structures of $1 \mathrm{D}$ graphene wire or the so-called nanoribbons (GNR) where the wire edge shape has early attracted attention. ${ }^{16}$ A recent study ${ }^{17}$ shows similar necklacelike electron dispersion branches [Fig. 4(b) in Ref. 17] separated by mini-band-gap stripes in a GNR whose zigzag edges are reconstructed to form a $0.49-\mathrm{nm}$-periodic corrugation. This specific edge-induced 1D DOS modulation, not highlighted by the authors, is absent in the band structure of a standard zigzag GNR deprived of minigaps [Fig. 4(a) in Ref. 17]. Undulated $\mathrm{GNR}^{18}$ may offer similar features resulting in large conductivity modulations.

Our fabricated structures are $\sim 200-\mu \mathrm{m}$-long planar $\mathrm{PhC}$ wire waveguides in an active InP/InGaAsP/InP vertical heterostructure. The active layer is $118 \mathrm{~nm}$ thick and embeds six InGaAsP quantum wells (QW) with emission wavelength centered at $1.55 \mu \mathrm{m}$. The ten rows of triangular PhC lattice laterally confining the broad wire have a pitch $a=400 \mathrm{~nm}$ and a fill factor of $f=35 \%$. The air holes are defined by electron-beam (e-beam) lithography and deeply etched through the heterostructure using inductively coupled plasma reactive-ion etching (ICP-RIE). ${ }^{19}$ Wires of three different widths, namely, W21, W31, and W41 corresponding to 21, 31 , and 41 missing rows in the $\Gamma K$ direction, were fabricated at a tilt angle of $30^{\circ}$ and cleaved at a $\sim 200 \mu \mathrm{m}$ length, as shown in the scanning electron microscopy (SEM) images in Figs. 3(a)-3(c). 
(a)

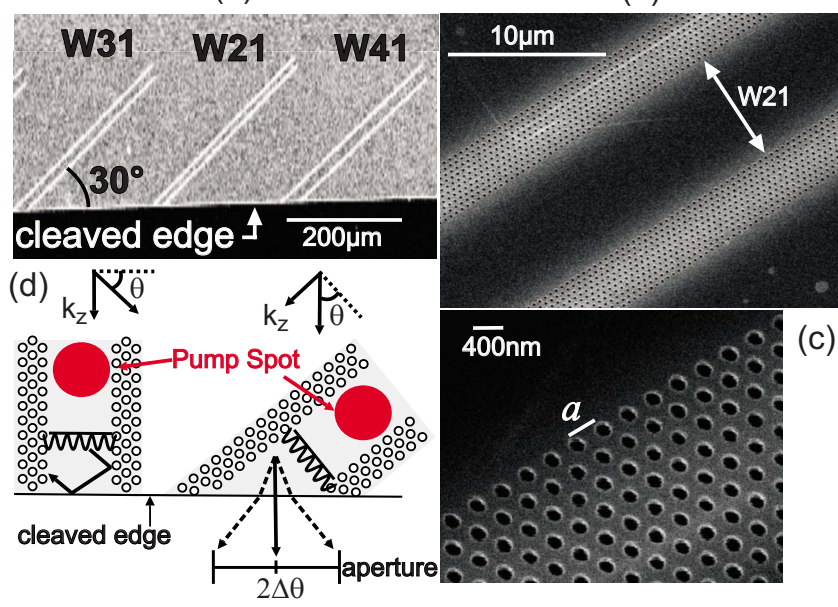

FIG. 3. (Color online) [(a)-(c)] Micrographs of fabricated waveguides. (d) Waveguides are tilted to observe higher-order modes. The $2 \mathrm{D} \mathrm{PhC}$ has a lattice of period $a=400 \mathrm{~nm}$.

The reason for such a tilt is apparent from Fig. 3(d). The necklace branches of the band diagram correspond to HOMs that remain trapped inside the waveguide due to TIR. Tilting the waveguides at a certain angle with respect to the cleaved edge allows these modes to be observed by the external detection apparatus. The choice of the tilt angle depends on the wire core index and the region of $k$ space to be observed. Given an effective index of InP heterostructure of 3.21, the critical angle at the dielectric-air interface is $18.1^{\circ}$ with the normal to the interface. However, the collection objective of numerical aperture 0.45 reduces the effectively observed light cone inside the substrate to a half-angle $\Delta \theta$ of $9^{\circ}$. The $30^{\circ}$ waveguide tilt places the modes traveling at $\theta=30^{\circ}$ inside the waveguide at the center of the aperture thus fixing the central wave vector $k_{\mathrm{z}}=2 n u \sin \theta$ of the probed region. The half angles provide an observed $k_{\mathrm{z}}$ range of $\Delta k$ $=2 n u[\sin (\theta+\Delta \theta)-\sin (\theta-\Delta \theta)]$. This experimental window $k_{\mathrm{z}}-u$ is shown as the unshaded region in Fig. 2. Different guide widths will not change this window but only the mode spacing seen in it.

The broad corrugated photonic wires are characterized by optical pumping using a 10-mW-red laser (664 nm). As shown in Fig. 3(d) the round pump spot locally excites the embedded QWs inside the waveguide at a certain distance from the cleaved edge. The guided photoluminescence (PL) intensity is collected by an objective at the cleaved facet and directed to a camera-fiber pickup. ${ }^{20}$ The InGaAs camera has a cutoff at approximately $u \approx 0.24(\lambda \approx 1700 \mathrm{~nm})$. A polarizer is inserted to allow selective measurements of TE and TM spectra. This setup permits us to scan the position of the source along the $\sim 200-\mu \mathrm{m}$-long guides and evaluate its effect on the SpE spectra.

The experimental spectra for the three waveguides along with their calculated DOS are presented in Fig. 4. The dips correspond to the minigap stripes and the humps to the necklace dispersion branches. The observed FSR of the spectral modulation, in both TE and TM polarizations, corresponds to the FSR of the dispersion branches at the first BZ edge [see Figs. 2 and 5(a)]. This FSR is less than that of the branches

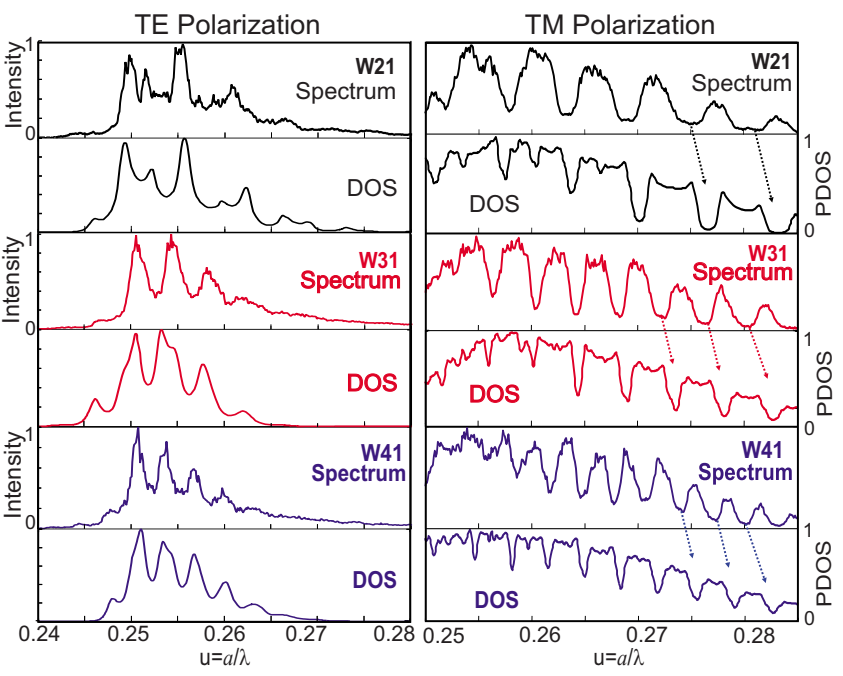

FIG. 4. (Color online) Experimental PL spectra of SpE from broad $\mathrm{PhC}$ waveguides and their corresponding DOS in the probed $k_{\mathrm{z}}-u$ window. The PL intensity is normalized.

at $k_{\mathrm{z}}=0$ (Ref. 11), thus ruling out any relation between the observed spectra and the $k_{\mathrm{z}}=0$ modes. For the TE polarization, the dips are wider than the humps in W21 but gradually narrow down in $W 31$ and $W 41$ because of the hyperbolic band curvature in the experimental $k_{\mathrm{z}}-u$ window. The cutoff of the InGaAs detector results in an apparently steeper decay in TE spectra at $u \approx 0.245$. The TM polarization spectra show

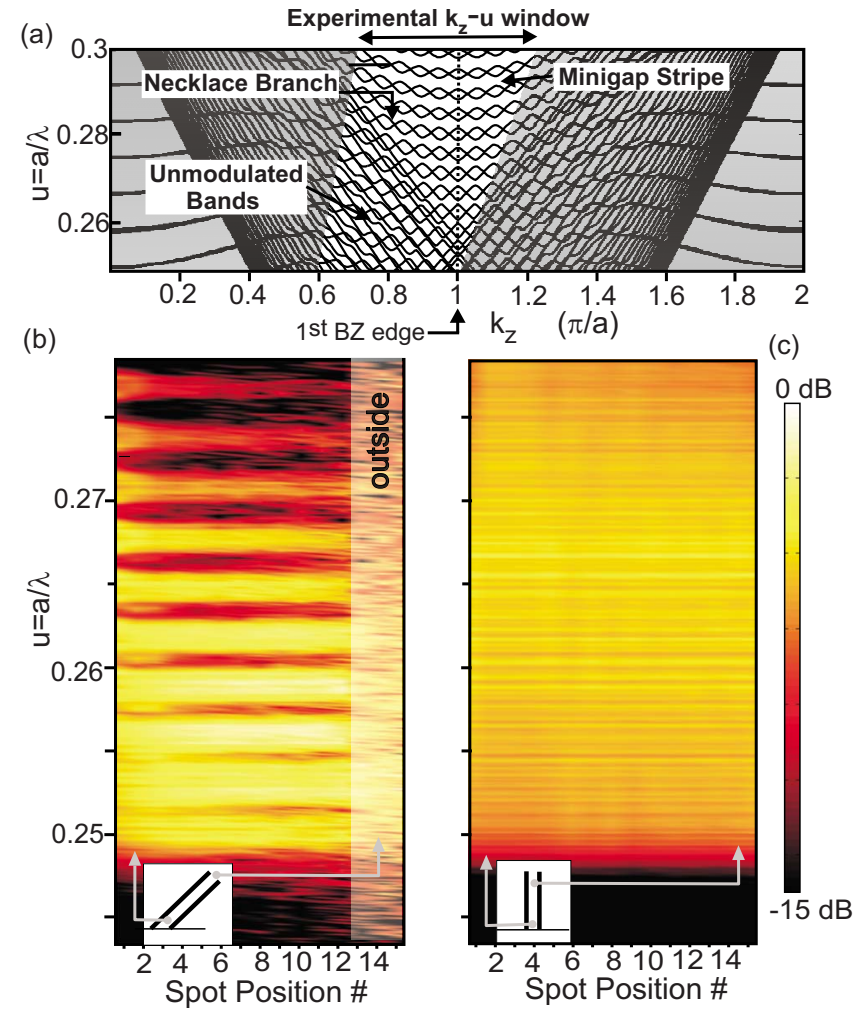

FIG. 5. (Color online) (a) Dispersion diagram of a W31 waveguide for TM polarization. The unshaded region is the experimental $k_{\mathrm{z}}-u$ window. (b) Position scan of the pump spot for a tilted and (c) a nontilted $W 31$ waveguide in TM polarization. 
deeper modulations that retain their contrast over a wider frequency range than their TE counterparts. We attribute this to the lower TM (re)absorption by QWs at high energies as well as to the lower scattering losses. ${ }^{21}$ The flat-top appearance of the humps in TM spectra (especially in W21 and W31) is due to the larger width of their dispersion branches as compared to TE polarization. Also observed in the TM spectra is the narrowing of the dips toward lower frequencies. A close examination of the TM band diagram [Fig. 5(a)] does reveal a trend of decreasing minigap stripe width toward lower frequencies, further blurred by unmodulated bands.

In order to trace back the observed spectra to the intrinsic waveguide DOS as in Ref. 4, we calculate the 1D DOS from the dispersion diagram of the corresponding waveguides restricting ourselves to the $k_{\mathrm{z}}-u$ window defined by the waveguide tilt and the collection setup. To take the finite photon lifetime into account, we weigh an $m$ th band $\delta\left[u-u_{m}(\theta)\right]$ in the window with Lorentzians $L\left(u_{\theta}\right)=1 /\left[1+\left(u-u_{m}(\theta)\right)^{2} / \Delta u\right]$. Since the spectrum lies across the fundamental electronic QW transition, $\Delta u$ depends on $u$ especially for TE. We thus used a smoothed step function starting from a small value $\left(2 \times 10^{-4}\right)$ at $u=0.24$ to a large value $\left(1 \times 10^{-3}\right)$ following a realistic Fermi-Dirac distribution. The DOS at each frequency is then the sum of Lorentzians for all bands with a fine enough $k_{\mathrm{z}}$ discretization. To mimic the $\theta$ dependence of the collection setup, the collection efficiency is approximated by a semicircular profile $c(\theta)$ around the central $\theta$ value. The QW spontaneous emission envelope $\operatorname{SpE}_{\mathrm{QW}}(u)$ is modeled as an asymmetrical Gaussian (red curve in Fig. 2). The resulting integrated partial DOS (PDOS) reads

$$
\operatorname{PDOS}=\left[\int_{\theta-\Delta \theta}^{\theta+\Delta \theta} c(\theta) L\left(u_{\theta}\right) d \theta\right] \operatorname{SpE}_{\mathrm{QW}}(u),
$$

where the Lorentzian plays the role of the DOS sum, $\int L\left(u_{\theta}\right) d \theta \equiv \int L\left(u_{k}\right) d k \equiv \Sigma_{k} \delta\left(E_{k}-E\right)$. As it appears in Fig. 4, Eq. (1) is in excellent agreement with the observed spectra for all waveguides. The material dispersion was not taken into account in the calculations. This explains the slight deviation of the calculated PDOS toward shorter wavelengths (higher $u$ ) as pointed out by dotted arrows in TM polarization case.

To substantiate the fact that the observed experimental spectra indeed arise from the $\mathrm{SpE}$ regime of a source inside the wire and not from the mere modulated transmission, we perform a position scan of the pump spot for both a tilted and a nontilted $W 31$ waveguide in TM polarization. Figure 5 shows the experimental map for a scan of 15 spectra for positions starting from close to the cleaved edge inside the waveguide. The nontilted waveguide in Fig. 5(c) shows no modulation at all. For the tilted guide, however, strong spectral modulation is observed as long as the pump spot remains inside the waveguide, as shown in Fig. 5(b). Near the entrance and out of the waveguide we enter a transmission regime and the modulation loses its contrast. This is a clue to the issue of distinguishing transmission vs $\mathrm{SpE}$ effects as discussed in Fig. 1(c). For an on-axis source positioned outside the guide, there is a negligible power coupled to the HOM, hence our results. We did manage to couple to higherorder modes with an off-axis position and observed in transmission weaker modulations than those in Fig. 4, similar to Fig. 1(c). This data, not shown here, calls for a refined analysis. The other clue in favor of $\mathrm{SpE}$ effect is simply the uniformity of Fig. 5(b) map along the wire, outlining the local origin of the $\mathrm{SpE}$ modification as well as the advantage of our scheme to excite the HOMs from inside.

To summarize, in this Brief Report we have experimentally evidenced the modulation of the photon DOS inherent to a periodically corrugated broad wire. The minigap stripes that cause the dispersion bands to appear like necklaces could not be detected in other more standard geometries. The large fraction of $k$ space involved suggests a useful impact in photonic applications where the efficiency of coupling from internal sources to external optics is an issue. The possibility to increase the minigap width and thus to squeeze the necklace bands to thin stripes offers exciting perspectives for slow-light studies, ${ }^{15}$ as well as for $\mathrm{SpE}$ and gain enhancements in a number of fundamental applications.

The authors are grateful to R. Brenot from Alcatel Thales III-V Laboratories for providing the samples, and we acknowledge the support of the European project FUNFOX.
${ }^{1} \mathrm{~S}$. T. Ho et al., in Optical Processes in Microcavities, edited by R. K. Chang and A. J. Campillo (World Scientific, Singapore, 1996), p. 339.

${ }^{2}$ S. Olivier et al., Phys. Rev. B 63, 113311 (2001).

${ }^{3}$ V. S. C. Manga Rao and S. Hughes, Phys. Rev. B 75, 205437 (2007).

${ }^{4}$ E. Viasnoff-Schwoob et al., Phys. Rev. Lett. 95, 183901 (2005).

${ }^{5}$ T. Lund-Hansen et al., Phys. Rev. Lett. 101, 113903 (2008).

${ }^{6}$ E. Schwoob et al., Opt. Express 12, 1569 (2004).

${ }^{7}$ H. Gersen et al., Phys. Rev. Lett. 94, 073903 (2005).

${ }^{8}$ V. S. C. Manga Rao and S. Hughes, Phys. Rev. Lett. 99, 193901 (2007).

${ }^{9}$ H. H. Tao et al., Phys. Rev. B 74, 205111 (2006).

${ }^{10}$ L. Martinelli et al., J. Lightwave Technol. 25, 2385 (2007).
${ }^{11}$ O. Khayam et al., Appl. Phys. Lett. 91, 041111 (2007).

${ }^{12}$ T. Makino, IEEE J. Quantum Electron. 29, 14 (1993).

${ }^{13}$ H. Benisty, J. Appl. Phys. 79, 7483 (1996).

${ }^{14}$ The dispersion branch obeys the hyperbolic relation given by $\omega \cong \frac{c}{n}\left(k_{\mathrm{z}}^{2}+4 m^{2} \pi^{2} / 3 W N^{2} a^{2}\right)^{1 / 2}$, where $m$ is the branch number. The derivation of the minigap stripe hyperbola shall be published elsewhere.

${ }^{15}$ H. Kurt et al., J. Opt. Soc. Am. B 25, C1 (2008).

${ }^{16}$ K. Nakada et al., Phys. Rev. B 54, 17954 (1996).

${ }^{17}$ Pekka Koskinen et al., Phys. Rev. Lett. 101, 115502 (2008).

${ }^{18}$ A. Isacsson et al., Phys. Rev. B 77, 035423 (2008).

${ }^{19}$ F. Pommereau et al., J. Appl. Phys. 95, 2242 (2004).

${ }^{20}$ R. Ferrini et al., IEEE J. Quantum Electron. 38, 786 (2002).

${ }^{21}$ P. I. Borel et al., Opt. Express 11, 1757 (2003). 